\title{
Not only headache: higher degree of sexual pain symptoms among migraine sufferers
}

\author{
Gal Ifergane $\cdot$ Itzhak Z. Ben-Zion • \\ Ygal Plakht $\cdot$ Keren Regev $\cdot$ Itzhak Wirguin
}

Received: 9 December 2007 / Accepted: 12 February 2008/Published online: 4 March 2008

(C) Springer-Verlag 2008

\begin{abstract}
Chronic illness and chronic pain can have profound negative effects on relationship and sexual satisfaction, yet the influence of migraine on sexuality has not been previously evaluated. To assess sexual functions in subjects with migraine compared to those with no migraine. We evaluated female university students using the Israeli sexual behavior inventory (ISBI). Migraine was diagnosed according to self-reported symptoms according to the IHS criteria. Several dimensions of female sexuality-desire, orgasm, sexual avoidance, interpersonal sexual relationship, health influence, satisfaction and pain were evaluated using a structured questionnaire. Thirty-three (23.9\%) of the participants met the IHS criteria for episodic migraine with and without aura. Sexual activity, desire, orgasm and satisfaction from sexual life did not differ significantly between migraine sufferers and non-sufferers. Migraine patients reported lower ISBI scores, higher health influence on sexual life, higher levels of sexual pain and lower sexual satisfaction. Migraine negatively affected the sexual life of sufferers. Sexual pain disorder
\end{abstract}

G. Ifergane $(\square) \cdot$ K. Regev $\cdot$ I. Wirguin

Headache Clinic, Department of Neurology, Soroka Medical

Center, Ben-Gurion University of the Negev, P. O. Box 151,

Beer-Sheva, Israel

e-mail: galif@clalit.org.il

\section{Z. Ben-Zion}

Sexual Dysfunction Clinic, Department of Psychiatry,

Soroka Medical Center, Ben-Gurion University

of the Negev, Beer-Sheva, Israel

\section{Y. Plakht}

Leon and Matilda Recanati School for Community Health Professions, Soroka Medical Center, Ben-Gurion University of the Negev, Beer-Sheva, Israel is more common among migraine sufferers compared to non-migraineurs.

Keywords Migraine $\cdot$ Sexuality $\cdot$ Sexual pain disorder

\section{Introduction}

Chronic illness [1] and chronic pain [2] can have profound negative effects on relationship and sexual satisfaction of both patients and partners. The effects of chronic illness on sexuality are multifactorial and have an impact on all phases of sexual response. Sexual desire, arousal and activity may be altered by illness through interference with the hormonal, vascular and neural integrity of the mind and the genitalia, as well as the effects and side effects of medications. Anxiety, fear, loss of self-esteem, grief and depression are strongly associated with chronic illness and may also impair sexual function.

Studies examining [3] the prevalence of sexual dysfunction, and the role of psychological variables, including quality of life, on sexual activity in patients with noncancer pain found that sexual dysfunction was common, and more frequently reported by those with greater disability and depression, shorter pain duration, and infrequent use of coping self-statements.

Although sexual dysfunction was commonly reported, subjects perceived it had less importance in quality of life than did other factors possibly because of adaptation to the impact of chronic illness on sexual function.

Migraine is an extremely prevalent disease: The lifetime prevalence of migraine in women is $33 \%$ and the 1-year prevalence of migraine in women is $25 \%$. In men, the lifetime prevalence is $13.3 \%$ and the 1 -year prevalence is $7.5 \%$. It is a chronic-episodic disease, most common in 
men and women between the ages of 15-55 years that can impair many aspects of life [4]. Still, its influence on sexuality has not yet been systematically evaluated. The aim of the present study was to compare the prevalence of sexual dysfunctions between the migraine sufferers and non-sufferers participants.

\section{Methods}

\section{Participants}

In the present cross-sectional study, we evaluated female students from the Ben-Gurion University of the Negev who agreed to participate in the study. All the participants were included after having received a detailed explanation on the purpose and design of the study. Previously published data or the researchers assumptions regarding pain and sexual function were not presented to the participants. The study was approved by the institutional ethical committee.

Female students were recruited from classrooms, in which a brief presentation of the study was given. Subjects who were pregnant, were not sexually active during the year prior to questionnaire administration, and subjects who suffered from any neurological disorder (other than headache) or sustained a head trauma during the last year, were excluded from the study.

The participants were requested to fill a questionnaire. Questionnaires were filled without the presence of an interviewer, and did not include any recognizable data to assure full anonymity of the collected data. All subjects completed the following questionnaires:

Demographics and background: Participants were asked to answer questions regarding their personal background (e.g., age, marital status, religion, extent of religious observance, height, weight etc.).

Headache history: The participants were asked to report whether they suffered from troubling headache and if so, to report headache characteristics and accompanying features, using a list of items (frequency, number of headache days/month, severity, aura symptoms, localization, quality, exacerbating factors, nausea, vomiting, sensitivity to light and sound, triggers etc.). Migraine diagnosis was based on the 2004 IHS criteria for migraine with and without aura [5].

Migraine disability assessment scale (MIDAS): The migraine disability assessment (MIDAS) scale was used to quantify headache-related disability [6]. MIDAS is a brief and reliable headache-specific tool, which captures headache-related disability. It consists of five questions relating to the influence of headache on everyday activities over the preceding 3 months.
The Israeli sexual behavior inventory (ISBI): The ISBI is a 33 items questionnaire which was primarily designed to assess the impact of sexual problems, chronic illness and disability on sexual functioning and experience [7].

The questionnaire was designed to evaluate several dimensions ("domains") of female sexuality-sexual desire, orgasm, sexual avoidance, interpersonal sexual relationship, health influence, satisfaction and pain, during the previous year. The participants rated their answers in a scale of 1-5 according to the rate of agreement with certain phrases or according to the incidence of experiencing phenomenon asked in the questionnaire.

This instrument was proved to be a reliable and valid self-report measure of sexual behavior.

\section{Data analysis}

Analysis was performed using SPSS Win (version13.0) software. The socio demographic and health related characteristics were presented as proportion for categorical variables and mean with standard deviation for continuous variables. The ISBI questions were mapped on the scale from 1 to 5 as the higher grade signed the lower dysfunction. The ISBI questions were combined into domains and the score for each domain was calculated as the sum of related questions grades. The socio demographic and health related characteristics were compared between the migraine sufferers and non-sufferers participants using Chi square test, Fisher's exact test and Student's $t$ test. The relationships between sexual behavior items, and migraine were examined using Mann-Whitney non-parametric test due to the small sample size in subgroups and discrete nature of the ISBI scores. The multivariable analysis for sexual behavior based on linear regression. A $p$ values of $<0.05$ (two tail) were defined as significant.

\section{Results}

Out of 172 candidates, 138 (about 80\%) agreed to participate in the study. Eleven subjects $(7.9 \%)$ met the exclusion criteria due to sexual inactivity during the last year. The rest, 127 participants were included into the study. The median age of the subjects was 25 years. Most of them were unmarried, but had a regular sexual partner. In addition, most of them were secular, heterosexual and used oral contraception.

Thirty-two $(25.2 \%)$ of the participants met the IHS criteria for episodic migraine with or without aura. The characteristics of the study population with and without migraine are presented in Table 1. Migraine sufferers did not differ significantly from non-sufferers in any of the 
Table 1 Study population characteristics

\begin{tabular}{|c|c|c|c|c|}
\hline Characteristic & Total population $(n=127)$ & Migraine $(n=32)$ & Non-migraine $(n=95)$ & $p$ \\
\hline Age, mean (SD) & $25.2(3.4)$ & $26.0(4.3)$ & $24.9(3.1)$ & 0.146 \\
\hline Married, \% & 25 & 25 & 25 & 1 \\
\hline Presence of children, $\%$ & 8 & 6.5 & 8.5 & 1 \\
\hline Presence of regular sexual partner, $\%$ & 76.4 & 67.7 & 79.3 & 0.188 \\
\hline BMI, mean (SD) & $21.8(3.0)$ & $21.3(2.4)$ & $22.0(3.2)$ & 0.323 \\
\hline \multicolumn{5}{|l|}{ Sexual orientation } \\
\hline Homosexual, \% & 2.5 & 0 & 3.4 & \multirow[t]{2}{*}{0.596} \\
\hline Bisexual, \% & 3.4 & 3.3 & 3.4 & \\
\hline \multicolumn{5}{|l|}{ Religiousness } \\
\hline Traditional, $\%$ & 5.7 & 6.5 & 5.4 & \multirow[t]{2}{*}{0.587} \\
\hline Orthodox, \% & 2.4 & 0 & 3.3 & \\
\hline \multicolumn{5}{|l|}{ Contraception } \\
\hline Oral, \% & 72.9 & 79.3 & 70.8 & \multirow[t]{2}{*}{0.495} \\
\hline Condom, \% & 18.6 & 17.2 & 19.1 & \\
\hline
\end{tabular}

Comparison between migraine and non-migraine subject

demographic measures, such as age, marital status, regular partner, and sexual orientation, nor by number of children, $\mathrm{BMI}$, religion, religiousness and contraceptive use.

Migraine features among migraine participants are summarized in Table 2. Aura symptoms were reported by $13(40.6 \%)$ of the patients. Only eight $(24.2 \%)$ of them had a previous physician diagnosis of migraine, and only two (6\%) were using prophylactic anti migraine medications.

Levels of sexual activity, sexual desire, ability to experience orgasm and general satisfaction from sexual life did not differ significantly between migraine and nonmigraine subjects. Nevertheless, migraine patients reported

Table 2 Headache characteristics among migraine suffering subjects

\begin{tabular}{ll}
\hline Characteristic & Migraine subjects $(n=32)$ \\
\hline Aura, \% & 39.4 \\
Headache features & 78.8 \\
Unilateral, \% & 87.9 \\
Throbbing, \% & 90.9 \\
Moderate to severe intensity, \% & 90.9 \\
Exacerbated by activity, \% & \\
Accompanying features & 51.5 \\
Vomiting/nausea, \% & 90.9 \\
Phonophobia/photophobia, \% & \\
MIDAS & $12.5(14.5)$ \\
Mean (SD) & 39.4 \\
Minimal (0-5), \% & 21.2 \\
Mild (6-10), \% & 15.2 \\
Moderate (11-20), \% & 15.2 \\
Severe (>20), \% &
\end{tabular}

a greater degree of pain during intercourse and a higher frequency of fear of sexual intercourse and penetration and lower levels of satisfaction (Fig. 1). The total ISBI score among migraine sufferers was significantly lower than in non-sufferers (mean rank 49.5 vs. $68.9 ; p=0.01$ ). Multivariable analysis showed that migraine, absence of regular sexual partner, and self-reported religiousness (traditional or orthodox) was independently associated with lower ISBI scores. Among these predictors, migraine had the strongest relationship with the dependent variable (Table 3 ). The adjusted $R^{2}$ of this model was 0.17 .

The severity of migraine, as reflected by the MIDAS score, was found to be correlated to lower scores in the health domain $\left(r_{\mathrm{s}}=0.404 ; p=0.024\right)$. It was not associated with other domains of the score or with the total ISBI score.

\section{Discussion}

Female sexual dysfunction [8] is a general term used for various disorders of the sexual process in women affecting the various segments of the sexual response cycle: desire, arousal, orgasm and resolution. Inability or difficulty to enjoy any of these stages can interfere with sexual satisfaction and cause some degree of dysfunction. Female sexual dysfunctions can be accordingly subdivided in the DSM IV into desire, arousal, orgasmic and sexual pain disorders.

In the presented study, we attempted to evaluate sexual function and dysfunction among young women suffering from migraine compared to non-migraine sufferers, using the ISBI. 


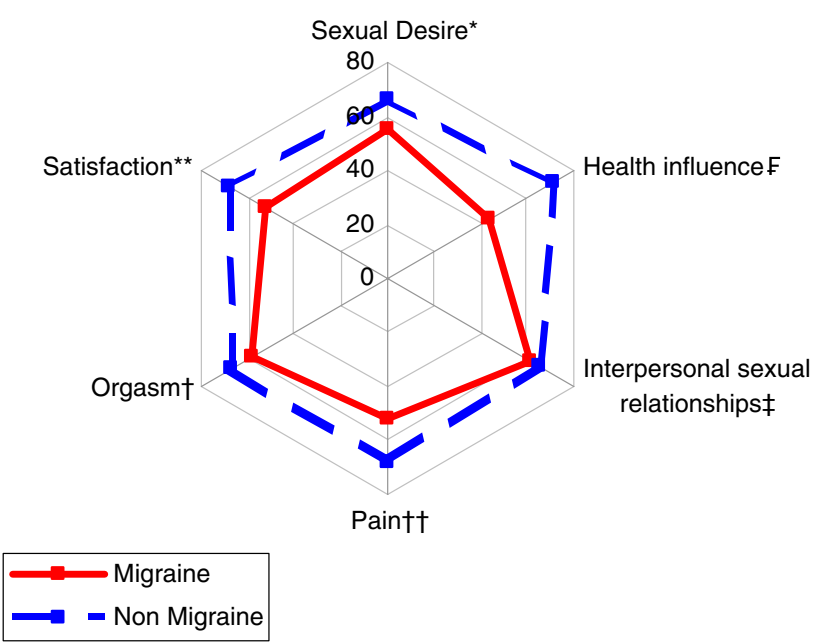

Fig. 1 Comparison of ISBI domain scores (mean ranks) between migraine sufferers and non-sufferers. * Sexual desire, $p=0.138$; ** satisfaction, $p=0.032 ; \dagger$ orgasm, $p=0.250$; $\dagger$ pain, $p=0.022$; interpersonal sexual relationships, $p=0.661 ; \mathrm{F}$ health influence, $p<0.001$

Sexual desire, orgasm and sexual interpersonal relations were similar among migraineurs compared to non-migraineurs, and where comparable to previous reports regarding Israeli women in this age group. Unlike a recent study [9], we did not find migraine patients to have higher degrees of sexual desire. Three domains were significantly impaired among migraine sufferers: health influence, sexual pain and satisfaction. Positive answers regarding fear of sex and avoidance of sexual intercourse were more prevalent among migraine patients.

As in other chronic pain conditions, health influence on sexual life is expected among migraine patients. Indeed its influence, reflected by higher scores in this domain, was found to be correlated with higher MIDAS scores.

It seems reasonable, that avoidance, fear of penetration and consequently lower satisfaction are associated with higher levels of sexually associated pain.

Sexual pain disorders $[8,10]$ fall into one of two categories: dyspareunia and vaginismus. Dyspareunia is genital pain experienced during or immediately after sexual intercourse. Vaginismus is the recurrent or persistent involuntary or subconscious voluntary spasm (or contraction) of the genital perineal muscles that surround the outer third of the vagina when the insertion of any object is attempted. Dyspareunia is estimated to affect $5-15 \%$ of women annually, according to the National Health and Social Life Survey, and vaginismus affects $15-17 \%$ of women presenting to a sex therapy clinic [8, 10, 11]. Our study questionnaire was not designed to evaluate the various aspects of sexual pain disorder, and this subject should be addressed in future studies.

A possible explanation for the high prevalence of sexual pain disorder among migraine females is sensitization of pain pathways $[10,12]$ often found in migraine patients (either as a cause or consequence). Clinical and experimental observations [13] on migraine suggest that a general hyper excitability could develop along nociceptive trigeminal neurons allowing the activation of descending pathways that facilitate pain processing or the suppression of pathways that slow down pain transmission. Slowly progressive dysfunction of central pain systems secondary to the repetition of attacks of the originating headache has been implicated in transforming the process from an episodic to a chronic condition. This concept of an irritable focus in the central nervous system is currently known as "central sensitization" and is a characteristic feature of visceral pain. Dyspareunia was found to be a co-morbid phenomenon with other pain syndromes [10, 11] (e.g. fibromyalgia), and to involve increased sensitivity to pain and touch [15]. These findings support the involvement of central sensitization in patients with sexual pain disorder.

It is also possible that migraineurs tend to be more attentive to somatic and pain symptoms.

Recurrent pain is often characterized by elevated painrelated fear and avoidance of behaviors which the headache sufferer believe will initiate or perpetuate the headache pain, a maladaptive process which serves to promote pain related disability [14]. This process might be the underlying reason for the increased fear of sexual intercourse and penetration among migraine patients in this study.

The present study was designed as a pilot study, exploring different aspects of female sexuality in migraine sufferers and non-sufferers. To avoid bias, the study was conducted in the community, and randomly selected the participating women. Female students were divided into migraine sufferers and non-sufferers. The evaluated population is not representative of the general population, but rather a homogenous population of young, educated, secular women belonging to a relatively high socioeconomic status. One has to take into account that only in such a population asking intimate sexual questions, and getting
Table 3 Predictors of ISBI total score-multivariable analysis

\begin{tabular}{lllll}
\hline Characteristic & B & Std. error & Beta & $p$ \\
\hline Migraine (yes vs. no) & -2.27 & 0.57 & -0.34 & $<0.001$ \\
Regular partner (no vs. yes) & -1.26 & 0.59 & -0.18 & 0.034 \\
Religiousness (traditional or orthodox vs. secular) & -1.98 & 0.89 & -0.19 & 0.028 \\
\hline
\end{tabular}


honest answers; is feasible. Compared to Israeli women in similar age group [6,7], our study population reported higher sexual desire, tended more to initiate sexual intercourse with their partner, and lower fear of intercourse and penetration. The interpretation of the findings should therefore be limited to this group of women only.

As the respondents were asked to report about only troubling headaches, only a small number of subjects reported headaches corresponding to IHS criteria of tension type headache. No information regarding tension headache can be concluded from this study and further investigation is required. Therefore, no conclusions can be drawn from the current study regarding patients with this type of headache.

Despite these limitations, our findings suggest that migraine affects the sexual life of women suffering from it. This effect is both severity dependent (as represented by the health influence domain) and independent (intercourse associated pain).

Future studies should be directed in exploring the incidence of sexual dysfunction in general and specifically sexual pain disorders in other migraine suffering populations, and in evaluating the exact nature of the sexual pain disorders. Findings of such studies may help in improving sexual related quality of life.

\section{References}

1. Schover LR, Jensen SB (1988) Sexuality and chronic illness-a comprehensive approach. The Guilford Press, New York
2. Ambler N, Williams AC, Hill P et al (2001) Sexual difficulties of chronic pain patients. Clin J Pain 17:138-145

3. Kwan KS, Roberts LJ, Swalm DM (2005) Sexual dysfunction and chronic pain: the role of psychological variables and impact on quality of life. Eur J Pain 9(6):643-652

4. Bigal ME, Lipton RB, Stewart WF (2004) The epidemiology and impact of migraine. Curr Neurol Neurosci Rep 4(2):98-104

5. Headache Classification Committee of the International Headache Society (2004) The international classification of headache disorders. Cephalalgia 24(suppl 1):1-160

6. Stewart WF, Lipton RB, Kolodner K, Liberman J, Sawyer J (1999) Reliability of the migraine disability assessment score in a population-based sample of headache sufferers. Cephalalgia 19:107-114

7. Kravetz S, Drory Y, Shaked A (1999) The Israeli sexual behavior inventory (ISBI): scale construction and preliminary validation. Sex Disabil 17(2):115-128

8. Laumann EO, Paik A, Rosen RC (1999) Sexual dysfunction in the United States: prevalence and predictors. JAMA 281:537-544

9. Houle TT, Dhingra LK, Remble TA, Rokicki LA, Penzien DB (2006) Not tonight, I have a headache? Headache 46(6):983-90

10. Binik YM, Meana M, Berkley K, Khalife S (1999) The sexual pain disorders: is the pain sexual or is the sex painful? Annu Rev Sex Res 10:210-235

11. Laumann EO, Paik A, Rosen RC (1999) Sexual dysfunction in the United States: prevalence and predictors. JAMA 281:537-544

12. Gordon AS, Panahian-Jand M, Mccomb F, Melegari C, Sharp S (2003) Characteristics of women with vulvar pain disorders: responses to a Web-based survey. J Sex Marital Ther 29(Suppl 1):45-58

13. Welch KM (2003) Contemporary concepts of migraine pathogenesis. Neurology 28;61(8 Suppl 4):S2-8

14. Norton PJ, Asmundson GJ (2004) Anxiety sensitivity, fear, and avoidance behavior in headache pain. Pain 111(1-2):218-223

15. Pukall CF, Strigo IA, Binik YM, Amsel R, Khalife S, Bushnell MC (2005) Neural correlates of painful genital touch in women with vulvar vestibulitis syndrome. Pain 115(1-2):118-127 